\title{
Measuring and Comparing Local Strain Field and Crystal Rotation at the Microscopic Scale
}

\author{
Florent Bridier ${ }^{1}$, Jean-Charles Stinville ${ }^{1}$, Nicolas Vanderesse ${ }^{1}$, Marin Lagacé ${ }^{2}$, Philippe Bocher $^{1}$ \\ 1. École de technologie supérieure, Mechanical Engineering Department, Montreal, Quebec, Canada \\ 2. Institut de recherche d'Hydro-Québec, Varennes, Quebec, Canada
}

Measuring strain fields at microscopic scales is a constant challenge to better relate the local mechanical behavior with the complex and fine microstructures present within structural parts. Particularly, many damage mechanisms of polycrystalline metallic alloys involve the accumulation of plastic strain at the sub-grain level or around grain boundaries which leads to crack nucleation and final rupture [1]. Moreover, little experimental data of local strain and crystal rotation at the micrometric scale is available to validate crystal plasticity finite element simulations. The present work illustrates the potential combination of the measurement of local microscopic in-plane strains and local crystal orientation during in-situ deformation test in the scanning electron microscope (SEM).

Using a Kammrath \& Weiss 5000N tension/compression stage within a Hitachi SU70 Schottky-SEM, in-situ tensile tests were performed on a micro-specimen of $316 \mathrm{~L}$ stainless steel (average grain size $\sim 50$ $\mu \mathrm{m})$. The surface of the specimen was etched in order to produce a nanometric speckle pattern which is favorable for digital image correlation without disturbing EBSD measurements. The loading machine was tilted to $50^{\circ}$ within the SEM chamber. The specimen itself was tilted to $20^{\circ}$ relative to the machine, leading to a specimen surface tilted to $70^{\circ}$ (Fig. 1), which enables to perform simultaneous EBSD analysis. The high resolution images were obtained using the lower fore-scatter detectors associated with an Oxford F+ EBSD detector. The corresponding EBSD maps were taken with a step size of $0.25 \mu \mathrm{m}$. Prior to deformation, initial images and EBSD maps were taken at both x300 and x1000 magnifications. During the tensile test, 9 interruptions at various stress levels were applied in order to acquire both SEM images at the maximum load and after elastic unloading and EBSD maps only after unloading.

The progressive strain fields at the microscopic scale were obtained using both a digital image correlation software to extract high resolution displacement field and a companion application to calculate the corresponding strain fields on the basis of isoparametric finite element formalism. These two applications were developed and implemented by the authors. As illustrated in Fig. 2, the progressive strain localization at the scale of metallurgic grains could be obtained. Moreover, the progressive activation of slip bands and related strain localization are noticeable and can be related to the surrounding microstructural features (grain/twin boundaries).

In parallel, cross-correlation analyzes of EBSD maps were performed to quantify the progressive local crystal rotation relative to the original crystal structure before deformation. To this matter, a computer program was written to obtain quantitative information about the local rotation, i.e., crystal orientation change, due to the plastic deformation. By the use of the common angle-rotation axis representation, the program correlates the pixels of two EBSD maps corresponding to the un-deformed and the deformed stage, respectively. It automatically provides the local crystal misorientation between any point of the EBSD map and the average crystal orientation of the un-deformed corresponding grain (Fig. 2). The average grain orientation was particularly obtained by the representation of orientations in the form of quaternions and the demonstrated calculation of their arithmetic average [2]. 
In conclusion, an experimental procedure has been developed and validated to systematically measure the local deformation behavior of polycrystalline materials at the micrometer scale. Both strain field and local crystal rotation field may be acquired and compared.

[1] F Bridier, P Villechaise and J Mendez, Acta Materialia 56 (2008), p. 3951.

[2] M Humbert et al, Journal of Applied Crystallography 29 (1996), p. 662.

[3] The authors acknowledge the Natural Sciences and Engineering Research Council of Canada and the MITACS Acceleration program for the financial support of this work.

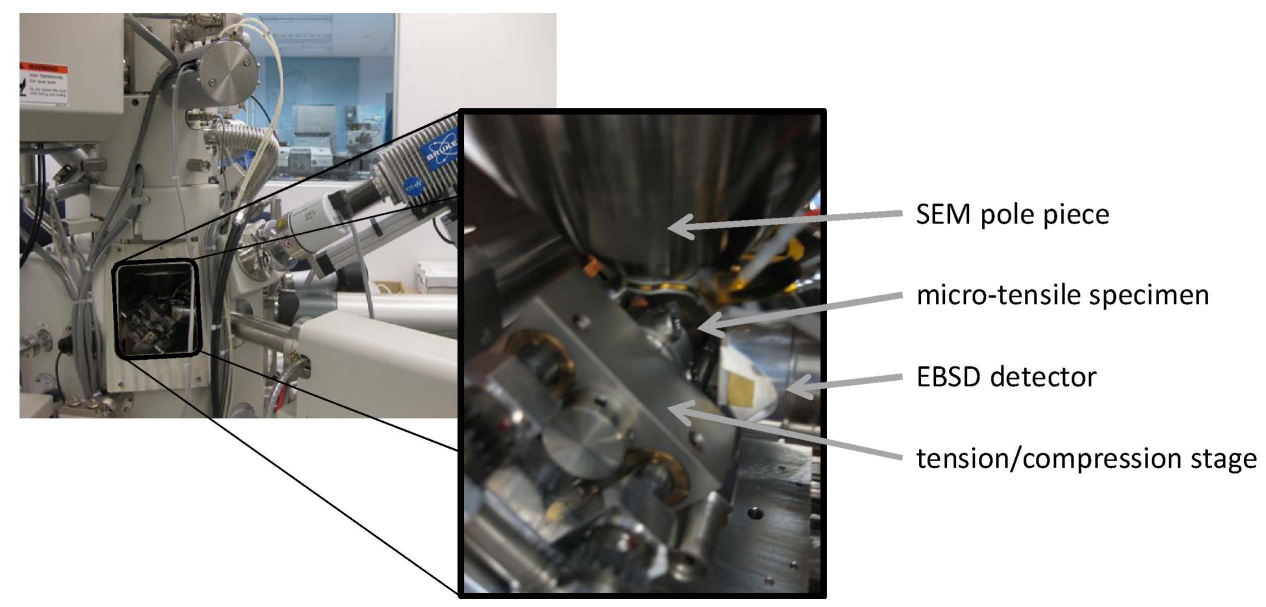

Figure 1. Experimental set up coupling in situ mechanical testing and EBSD measurement

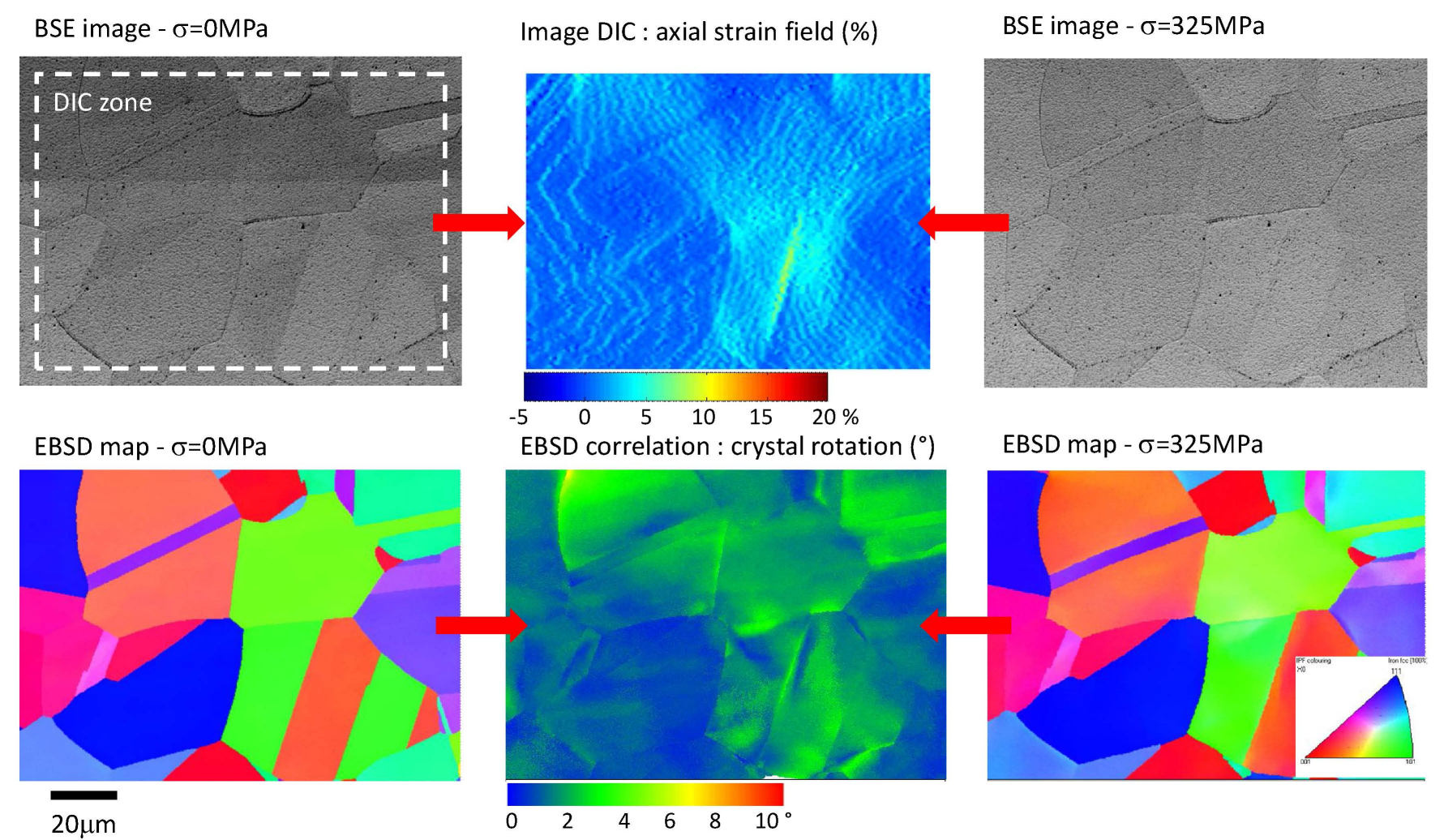

Figure 2. Local strain field and crystal rotation obtained at the $325 \mathrm{MPa}$ step for the same zone 